\title{
Bienestar y satisfacción de los médicos con el ejercicio de su profesión en un sistema de salud en proceso de reforma
}

\author{
Juan Pablo Jiménez \\ Comfort and satisfaction of \\ physicians with their medical \\ practice in a reforming health system
}

The subjective wellbeing of physicians and the degree of dissatisfaction with their practice is nowadays an important issue in English speaking journals. There is an international perception of a growing and deepening crisis in health systems that is affecting the professional and personal life of physicians. A multidisciplinary group, from two main Universities in Chile, has been formed to investigate this situation locally. The results of this investigation will be published in successive issues of Revista Médica de Chile (Rev Méd Chile 2004; 132: 635-6).

(Key Words: Health care quality, access, and evaluation; Health care reform; Health care surveys; Practice guidelines)

Recibido el 25 de noviembre, 2003. Aceptado el 18 de marzo, 2004.

Departamento de Psiquiatría y Salud Mental Oriente, Facultad de Medicina, Universidad de Chile, Santiago de Chile.

$\mathrm{E}^{\mathrm{a}}$ nuestro país se está implementando una ambiciosa reforma en el sistema de salud, como el cambio estructural más profundo en el sector, desde la creación del Servicio Nacional de Salud, a comienzos de la década de los cincuenta. Tanto en Chile, como internacionalmente, existe la percepción generalizada que el sistema de salud está en crisis, como consecuencia de los cambios macro-sociales, de los vertiginosos cambios tecnológicos relacionados con el diagnóstico y la terapéutica, y de los ocurridos en la estructura, gestión y financiamiento de los servicios de salud. Estos cambios han transformado acelerada-

Correspondencia a: Juan Pablo Jiménez. Salvador 486, Providencia. Fax: 2748855. E-mail: jijmenez@med.uchile.cl mente el ejercicio de la profesión médica, agregándole nuevas presiones, pero también oportunidades.

Existen diversos estudios sobre las variables económicas del problema y sobre la insatisfacción de los usuarios con el sistema. Los médicos, sin embargo, como el gremio más conspicuo y representativo dentro de las profesiones de prestación de servicios sanitarios, recién empiezan a ser estudiados, fundamentalmente en algunos países desarrollados, en relación con el impacto que esta crisis tiene en su subjetividad; es decir, en su compromiso vocacional, satisfacción laboral, en su identidad profesional $y$, desde luego, en el efecto de todo esto en su rendimiento laboral, salud física y emocional, vida personal y familiar 
(calidad de vida). Entre nosotros, ha sido fundamentalmente el gremio médico quien ha hecho pública la inquietud entre sus afiliados. Con todo, el malestar que el gremio ha puesto de manifiesto, generalmente se basa en información anecdótica, recogida a través del contacto de sus dirigentes con las bases. Por otra parte, al revisar las principales publicaciones médicas de habla inglesa de los últimos cinco años, podrá constatarse un creciente interés en el tema, que se traduce en un corpus creciente de investigaciones sistemáticas sobre distintos aspectos de la subjetividad de los médicos.

Este panorama nos ha motivado a inaugurar una línea de investigación nacional sobre este tópico. Hemos formado un grupo de investigación, surgido del Departamento de Psiquiatría y Salud Mental Oriente de la Universidad de Chile y del Departamento de Salud Pública de la Pontificia Universidad Católica de Chile*, con el propósito de estudiar sistemáticamente la subjetividad de los médicos chilenos, sobre la base de una muestra representativa de la población médica de la región metropolitana. Los resultados de esta investigación serán comparados con estudios simultáneos realizados en Buenos Aires y Montevideo, como parte de un proyecto internacional multicéntrico.

Durante el año 2002, nuestro grupo revisó el estado de la discusión, según se refleja en las publicaciones internacionales y llevó a cabo un estudio cualitativo exploratorio en diferentes grupos de médicos de la región metropolitana. A lo largo de este proceso nos ha quedado claro que el campo de la subjetividad es complejo y difícil de definir con precisión. Algunas de las principales dimensiones que lo componen son las siguientes:

- El bienestar subjetivo versus la percepción de malestar;

- La satisfacción versus la insatisfacción con el ejercicio de la profesión;

- La relación entre la calidad del ejercicio profesional -donde se incluye la calidad de la relación que se establece con los pacientes- y calidad de vida;

- La calidad de las relaciones íntimas, es decir, la vida en pareja, en familia y la relación con las amistades;

- El mundo valórico del médico, que incluye variables espirituales como la adhesión a la «ocación» de servicio y, finalmente,

- La capacidad de autocuidado de los médicos, que se relaciona con los grados y tipos prevalentes de patología física y mental (por ejemplo, estrés y ঝburnout»entre los médicos). En sucesivos artículos revisaremos el estado actual de la discusión internacional y presentaremos los principales hallazgos del estudio cualitativo realizado.

* El grupo investigador está formado por los Dres. Juan Pablo Jiménez, Julia Acuña y Claudio Fullerton, la socióloga Nina Horwitz y la psicóloga María Luz Bascuñán, de la Universidad de Chile, y por el Dr. Jorge Jiménez y el estadístico Luis Villarroel, de la Pontificia Universidad Católica de Chile. 